\title{
Inverse statistics and misperception of exponential growth
}

\author{
HAN TIMMERS and WILLEM A. WAGENAAR \\ Institute for Perception TNO, Soesterberg, The Netherlands
}

\begin{abstract}
Exponential growth presented by numerical series or graphs is grossly underestimated by human subjects. This misperception was considerably lessened by presenting decreasing functions; this conclusion holds for both numeric and graphic stimuli. In the numerical conditions, about $25 \%$ of the subjects performed according to the statistical norm. In contrast with previous results, considerable individual differences with respect to sensitivity for rate of growth were observed. This finding was interpreted in terms of task difficulty: Extrapolation of ascending series is too difficult a task to be discriminative. Extrapolation of descending series is much easier, and may therefore better discriminate among subjects.
\end{abstract}

Exponential growth presented by numerical series or graphs is grossly underestimated by human subjects. Recent research (Wagenaar \& Sagaria, 1975) showed that people take account of only a small proportion of the exponent when asked to intuitively extrapolate the function $y=e^{b x}$. The responses are well described by a simple model according to which people extrapolate as if the function were $y=\left(\alpha \mathrm{e}^{\beta b}\right)^{\mathrm{x}}$. For the majority of the subjects, the value of $\beta$ amounted to about 0.20 for numerical representations and 0.04 for graphical representations. The individual differences were largely due to the considerable variation of the value of $\alpha$.

An important objective of this research program was to present exponential growth processes in such a way that the average man in the street could understand what was going on.

One solution that might serve this aim is suggested by the work of Tversky and Kahneman (1973) on the availability heuristic. They demonstrated that the value of $1 \times 2 \times 3 \times \ldots 8$ is estimated much better when subjects are asked to extrapolate $8 \times 7$ $\times 6 \times \ldots 1$. The large initial products in the latter case are the more suggestive of the final result. In a similar way, one might guess that extrapolation of exponential series is easier when the series decrease, i.e., when the exponent is negative.

Examples of this kind of presentation are: square miles per individual as a measure of population increase, average elapsed time between two crimes as a measure of growing crime rates, liters of gasoline that one dollar buys as a measure of rising price levels. (It is not suggested that these processes always show exponential growth.) Some intuitive appreciation of this kind of presentation will be obtained by looking at the following exponential series.

H. Timmers is now at the Institute for Perception Research, Eindhoven, The Netherlands.

$\begin{array}{rrr}\text { Year } & \text { Index 1 } & \text { Index 2 } \\ 1971 & 3 & 22,026 \\ 1972 & 7 & 8,103 \\ 1973 & 20 & 2,981 \\ 1974 & 55 & 1,097 \\ 1975 & 148 & 403\end{array}$

The best predictions for the year 1980 are 22,026 (Index 1) and 3 (Index 2). Many readers will find it much more difficult to believe the first prediction than the second one.

The objective of the experiments reported here was to test the hypothesis that perception of exponential growth is much more accurate when decreasing series are presented; again, numerical and graphical representations will be compared. In the graphical case, however, a special problem presents itself, as demonstrated in Figure 1. The extrapolation task in Figure $1 \mathrm{~b}$ is somewhat meaningless to the subjects, since they cannot discriminate between various answers, whether they are extremely conservative (e.g., 148) or accurate (3). Therefore, the technique used in the graphical condition will be of a different kind. Subjects will be asked to pairwise compare two graphs, one ascending and one descending; the question to answer is always: Which of the two graphs will reach a certain level sooner?

\section{EXPERIMENT 1: NUMERIC STIMULI}

\section{Method}

Procedure. Samples of decreasing processes over the years 1971 to 1975 were presented to two groups of subjects, together with one of two alternative questions: If nothing will stop this decreasing trend, (Group 1) What is your prediction for 1980? (Group 2) When will the process reach . . . (a certain level)?

The general function describing the stimulus series was $y=a e^{b x}$ ( $y=$ index; $x=0,1, \ldots, 4=$ number of years since 1971). The 21 combinations of $a$ and $b$ used were: 

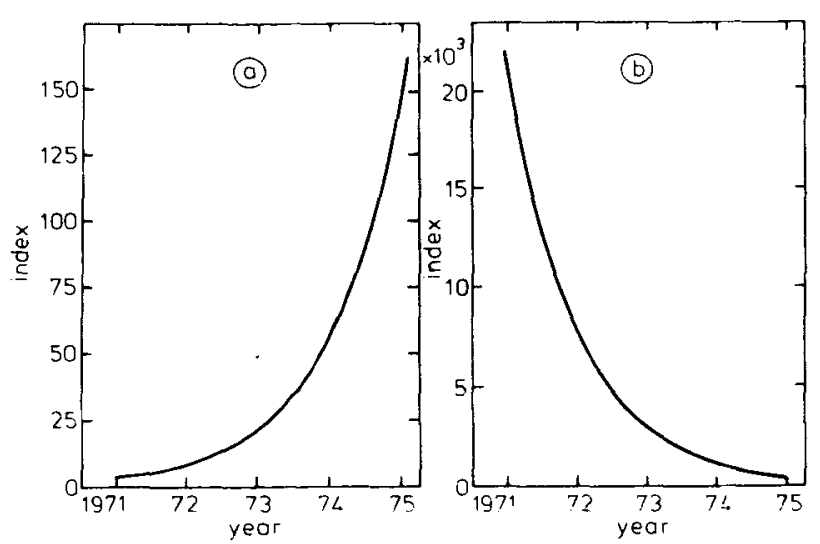

Figure 1. Ascending and descending representations of exponential growth. The exponent is 1.0 in both instances.

$$
\begin{aligned}
& a \approx 1,000 ; b=-0.1,-0.2, \ldots,-0.7 \\
& a \approx 25,000 ; b=-0.3,-0.4, \ldots,-0.9 \\
& a \approx 625,000 ; b=-0.5,-0.6, \ldots,-1.1
\end{aligned}
$$

A complete factorial combination of $\mathbf{a}$ and $\mathbf{b}$ was not used in order not to involve too small values of $y$.

The level indicated for Group 2 was always reached in 1980 according to normative extrapolation. All starting series were presented to both groups. The 21 problems were printed on successive pages of a booklet; the order of presentation was randomized among subjects. The instruction stressed that subjects should not attempt to apply formal mathematical rules; rather, they were asked to give their intuitive estimations. Subjects were allowed to spend $30 \mathrm{sec}$ at each problem; the experimenter indicated when they should turn to the next page.

Subjects. Thirty-five subjects, students of the State University of Utrecht, took part in this experiment. They were paid Dfl. 5,-for their participation.

\section{Results}

The results will be interpreted according to the mathematical model presented in the introduction.

Group 1. The responses of Group 1 can be described by:

$$
\hat{y}=a e^{4 b}\left(\alpha e^{\beta b}\right)^{5},
$$

with $\hat{y}=$ prediction for $1980 ; a e^{4 b}=$ last number of the starting series. From Equation 1, it follows that:

$$
\ln \hat{y}=\ln a+5 \ln \alpha+b(4+5 \beta)
$$

Thus, the model predicts that a plot of lnŷ vs. b should be linear with slope $(4+5 \beta)$ and intercept $(\ln a+5 \ln a)$. The plots of $\ln \hat{y}$ vs. $b$ are presented in Figure 2 for the first, second, and third quartiles of the response distribution. These quartiles need not represent typical subjects. The concordance among the rank ordering of subjects for the three levels of a (Siegel, 1956) were 0.57, 0.54, $0.47\left(\chi^{2}=136,129\right.$,
$112 ; \mathrm{p}<<.001$ in each case); this indicates that the subjects tended to retain their relative positions in all conditions, and that the quartile scores can be interpreted as the behavior of some representative subjects.

The linear relationship between $\ln \hat{y}$ and $b$, which is specified by the model, is indeed shown by the results in Figure 2. The variance accounted for by the linear components was never below $93 \%$. For individual subjects, the linear components explained $92 \%$ (median value), with a minimum of $35 \%$ for one subject. The most striking effect is that the best $25 \%$ of the subjects produced predictions according to the norm.

Values of $\alpha$ and $\beta$, estimated from the intercept and the slope, are presented in Table 1 . The individual differences were mainly found in $\beta$, in contrast with the results on the increasing number series (Wagenaar \& Sagaria, 1975); the values of $\alpha$ were close to 1.0 and almost equal for all subjects. The results in Table 1 reveal that only the subjects above the third quartile did not profit from the inverse presentation ( $\beta$ is still around 0.20 ). The absolute magnitude of the starting series (a-factor) did not change $\alpha$ and $\beta$ (Friedman test: $\chi^{2}=0.40$, $\mathrm{df}=$ 2 , n.s.; $\chi^{2}=2.80, \mathrm{df}=2$, n.s.). Still there is some effect of the a-factor: The variance of the raw responses tended to increase with $a$; for many subjects the relation between predictions and values of

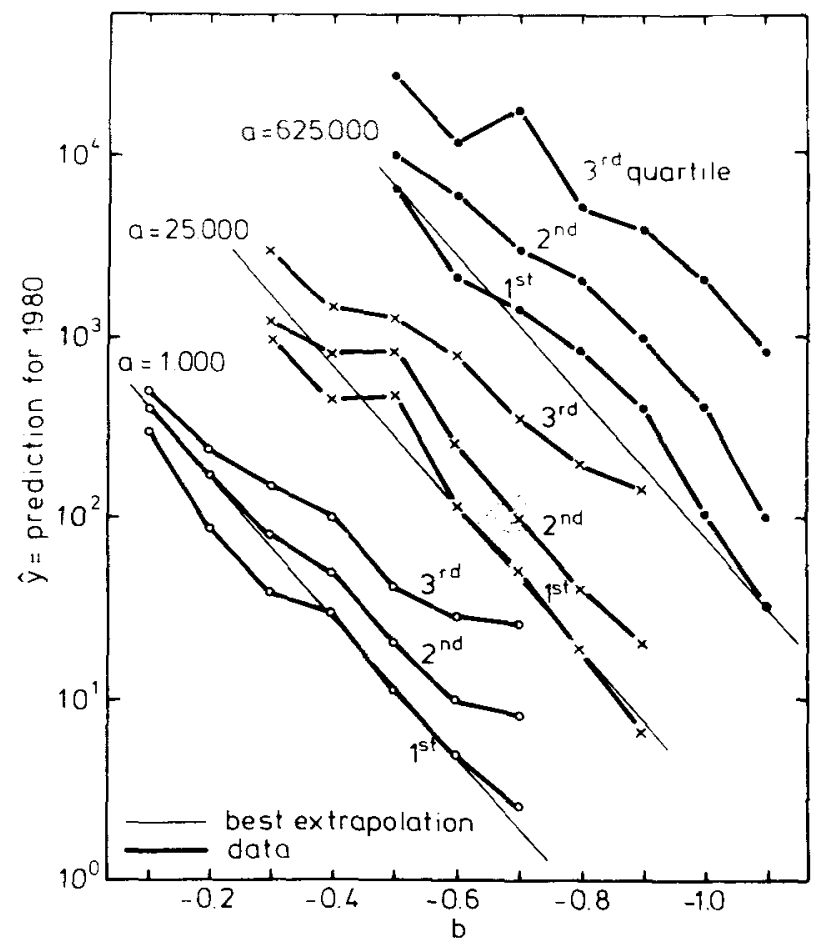

Figure 2. Results of Group 1. Starting numbers $y=a e^{b x}$ $(\mathbf{x}=\mathbf{0}, 1 \ldots, 4)$. 
Table 1

Results of Group 1

\begin{tabular}{cccc}
\hline a & Quartile & $\alpha$ & $\beta$ \\
\hline \multirow{3}{*}{1,000} & 1st & .87 & .72 \\
& 2nd & .92 & .54 \\
& 3rd & .93 & .23 \\
\multirow{2}{*}{25,000} & 1st & .93 & .89 \\
& 2nd & .92 & .65 \\
& 3rd & .89 & .22 \\
625,000 & 1st & .93 & .86 \\
& 2nd & .96 & .65 \\
& 3rd & .95 & .31 \\
\hline
\end{tabular}

$b$ tended to become less pronounced when a increased.

From Expression 2, it follows that plots of $\ln \hat{y}$ against Ina yield linear functions with unity slope. This prediction of the model is tested by the data collected from the stimulus series with $b=-0.5$, $-0.6,-0.7$, which all levels of a had in common. The slopes $(0.96,0.99,0.92$ for the median subjects) do not differ from unity to a significant degree $(\mathrm{t}=$ $0.41,0.07,0.75 ; \mathrm{df}=1$ ). These results also corroborate that the absolute magnitude of number does not affect the predictions in a systematic way.

Group 2. According to the model, the estimates of Group 2 can be described by:

$$
\hat{y}=1975+5 b /(\ln \alpha+\beta b) .
$$

This expression follows from equating $a e^{9 b}$ to $a e^{4 b}$ $\left(\alpha e^{\beta b}\right)^{x}$. Expression 3 can be rewritten as

$$
\frac{1}{\hat{y}-1975}=\frac{\ln \alpha}{5 b}+\frac{\beta}{5} \text {. }
$$

Consequently a plot of $1 / \hat{y}-1975$ vs. $1 / b$ should be linear with slope $\ln \alpha / 5$ and intercept $\beta / 5$.

When the values of $\alpha$ obtained with Group 1 are substituted in Formula 4, it follows that the expected differences between values of $\hat{y}$ in the various $b$ conditions are only minimal. For example, when $a=625,000$, the first quartile prediction for $b=$ -0.1 and $b=-0.7$ would both be close to 1980 . Therefore, the data do not lend themselves for a test of the model. It is worth noticing, however, that out of 63 scores ( 3 quartiles $\times 21$ combinations of a and b) 61 are below the predicted values, which indicates that the growth was perceived more accurately in this group $\left(\mathrm{p}_{\text {sign test }}<<.01\right)$. Even the third quartile subjects performed very well; for the three levels of a, their predictions were 1980, 1982, 1982.

\section{Discussion}

The most prominent result is, of course, the considerable reduction of underestimation, brought about by presentation of decreasing series. Some clarification is needed with respect to the values of $\alpha$ and $\beta$ in the various conditions. In the case of increasing series, good extrapolators differed from less successful subjects only with respect to values of $\alpha$; the effect of experience was also mainly reflected by an increase of $\alpha$ (Wagenaar \& Sagaria, 1975). Thus the picture emerges that extrapolation of increasing series was too difficult for all subjects; if it is true that subjects looked at differences between numbers rather than at ratios, it becomes clear that the distance up to the normatively correct response was very large compared to the range covered by the starting numbers. The only way of performing reasonably was by selecting a response range containing large numbers, more or less independent of the growth presented in the starting series. The values of $\alpha$ reflect this selection. Thus, the constancy of $\beta$ across subjects is interpreted as a floor effect due to extreme task difficulty. In the present experiment, the picture is quite different; the available range of responses was small compared to the range covered by the starting series, and moreover the range was strictly limited by zero. Not unlike Tversky and Kahneman's (1973) effect in computing $8 \times 7$ $\times \ldots \times 1$, the starting series lead the subjects right into the correct response range, and the responses may now reflect the accuracy of perceiving growth. An easy analogy presents itself: One cannot discriminate lifting power by requiring people to lift a 1,000-kg weight; lifting a $100-\mathrm{kg}$ weight, however, might be highly discriminative.

\section{EXPERIMENT 2: GRAPHIC STIMULI}

The results obtained thus far are not easily replicated with graphic stimuli, because of the problems mentioned in the introduction. Therefore, a paired comparisons paradigm had to be used. The subjects were asked to inspect a figure with one ascending and one descending curve. It was asked which of the two curves would first cross the (upper or lower) horizontal margin of the figure. The responses were analyzed by Guttman's scalogram analysis (Dawes, 1972).

\section{Method}

Stimulus materials. The stimuli were 72 graphs of $15 \times 45 \mathrm{~cm}$. Each graph contained two curves, one ascending and one descending (Figure 3). The abscissa of the graph represented values of $x$ ranging from $x=0$ to $x=16.7$; the ordinate ranged from $y=0$ to $y=1.0$. The graphs were grouped into two sets, $A$ and $B$. In set $A$, the ascending curves described functions of the general form $y=a e^{b x}$; the six values of $b$ were $0.1,0.2, \ldots$, 0.6. The value of a was chosen in such a way that $y=1.0$ for $x=9$ (i.e., $a=e^{-9 b}$ ). Thus, all ascending curves crossed the line $y=1.0$ at the same point. In principle, ascending and descending curves differ only with respect to the sign of $b$. In order to let all descending curves cross the line $y=0$ at $x=9$, at down- 


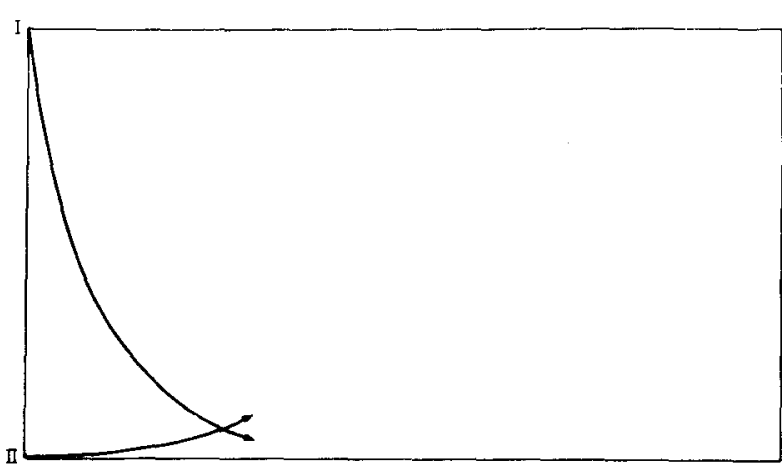

Figure 3. Example of stimuli used in Experiment 2; the exponents are +0.6 and -0.6 .

ward translation was applied by subtracting $e^{9 b}(b=-0.1$, $-0.2, \ldots,-0.6)$. Hence, the curves used for stimulus presentation are parts of the same curve, but located in such a way that the lines $y=0$ and $y=1.0$ are crossed at $x=9$. The formulas describing the actual stimulus materials are:

$$
y=e^{b(x-9)} \quad(b=0.1,0.2, \ldots, 0.6)
$$

and

$$
y=e^{b x}-e^{9 b} \quad(b=-0.1,-0.2, \ldots,-0.6) .
$$

The 36 graphs in set $A$ were obtained by a factorial combination of ascending and descending curves. The 36 graphs in set $B$ were obtained by mirroring the graphs in set $A$ around the line $\mathrm{Y}=0.5$. Thus, the ascending and descending curves are described respectively by:

$$
y=1-e^{b x}+e^{9 b} \quad(b=-0.1,-0.2, \ldots,-0.6)
$$

and

$$
y=1-e^{b(x-9)} \quad(b=0.1,0.2, \ldots, 0.6) .
$$

Set B was included in order to render dominance of curves with either positive or negative values of $b$ independent of the frequencies of the response alternatives (ascending, descending).

Procedure. For each subject, the 72 graphs were put in a different randomized order. The subjects worked through the file on 2 successive days. For each graph, they indicated which curve would cross the margin first. Writing or scribbling on the graphs was not permitted. No time limits were set; most subjects completed the task in less than $15 \mathrm{~min}$ a day. The subjects were run individually.

Subjects. The subjects were 27 students from the University of Utrecht. They took part in a larger experimental program, for which they were paid Dfl 50,- a day.

\section{Results}

Raw scores are presented in Table 2. The differences between set A and B were negligible $\left(\chi^{2}=0.29\right.$, $\mathrm{df}=36$ ). In total, 1,307 scores $(67 \%)$ preferred the curves with negative values of $b$. The raw scores are translated into a raw dominance matrix by substituting $\mathbf{0}$ for scores below 27 and 1 for scores above 27 . This matrix actually represents median responses to each pair of curves. Through rearrangement of rows and columns a pattern can be obtained that is perfectly triangular (Table 3). After interlocking the two scales, the result looks like Figure 4. The order of $-0.6,-0.4$, and -0.5 is arbitrary, as is the case with $0.3,0.6$, and 0.5 . The present order was chosen on the basis of row and column totals of raw scores.

The signed distances in Figure 4 correlate quite well with the raw scores: $r=0.82(z=4.79$, $p<.001$ ), which means that $66 \%$ of the variance is accounted for by the rank order of stimuli on this single dimension. About half of the remaining variance is introduced by stimulus -0.5 ; deletion of this stimulus from the data results in $r=0.90$.

\section{Discussion}

The results in Figure 4 show that generally a curve with negative $b$ is seen to cross the margin sooner than a curve with positive $b$. The effect is large for $b= \pm 0.6$ and \pm 0.5 ; the effect is moderate for $b= \pm 0.4$ and \pm 0.3 , while it is absent for $b= \pm 0.2$ and \pm 0.1 . These results can be explained by Tversky and Kahnemann's (1973) availability principle: The ascending curves are less representative of the final result of extrapolation, and the difference between ascending and descending curves increases with the absolute value of $b$. One might wonder whether this assertion is theoretically different from the hypothesis put forward by Jones (1977), namely that subjects fit low-grade polynomials to the stimulus data. In the extreme, they might extrapolate linearly, which would certainly provoke the present results. However, the results of Experiment 1 cannot be explained by linear extrapolation, since this would lead to overestimation of exponential growth. As a matter of fact, any polynomial fitted through the descending stimulus data would overestimate the growth function. On the other hand, it is intuitively appealing to hypothesize that subjects do weigh successive differences besides weighing ratios, as suggested by our model. A reconciliation of these views can be obtained when it is assumed that subjects intuitively estimate growth rate (the exponents $\beta b$ in Formula 1)

Table 2

Raw Scores in Experiment 2

\begin{tabular}{lllllll}
\hline & .1 & .2 & .3 & .4 & .5 & .6 \\
\hline-.1 & 35 & 21 & 30 & 21 & 39 & 42 \\
-.2 & 24 & 21 & 29 & 25 & 40 & 39 \\
-.3 & 31 & 24 & 40 & 38 & 43 & 39 \\
-.4 & 38 & 36 & 43 & 34 & 47 & 45 \\
-.5 & 31 & 35 & 28 & 40 & 42 & 34 \\
-.6 & 46 & 39 & 42 & 46 & 49 & 51 \\
\hline
\end{tabular}

Note-Each number indicates how often the curves with negative exponents were predicted to cross the margin first.

$$
\text { time to cross the frome }
$$

Figure 4. Interlocked scales for ascending and descending graphs. 
on the basis of successive differences in the stimulus series; larger differences provoke values of $\beta$ that are closer to 1.0 . The availability principle would operate through the estimation of growth rate, not through the extrapolation process itself.

Tentatively, the model could be applied on the present data. In that case, one would like to estimate the increase of $\beta$ brought about by taking negative values of $b$. The model asserts that the ascending curve (Formula 5) will cross the line $y=1.0$ when the last value shown to the subject $\left[\mathrm{e}^{\mathrm{b}(5-9)}\right]$ is multiplied $t$ times by the subjectively estimated multiplier $\left(\alpha \mathrm{e}^{\beta b}\right)$. Thus we obtain the expression $1.0=$ $\mathrm{e}^{b(-4)} \cdot\left(\alpha \mathrm{e}^{\beta b}\right)^{t}$. The line $y=1.0$ is crossed when $\mathrm{x}=\mathrm{t}+5$. After some rearrangement, we get $t=4 b /(\ln \alpha+\beta b)$. The same result is obtained for descending curves. This result means that the spread of stimuli with the same sign of $b$ is brought about by a value of $\alpha \neq 1$. In the case of $\alpha=1$, all values of $t$ would equal $4 / \beta$. In the following, we will use $\left[\alpha_{1}, \beta_{1}\right]$ when $b$ is positive, and $\left[\alpha_{2}, \beta_{2}\right]$ when $b$ is negative.

Now assume that an ascending function has a positive exponent, $b_{1}$, coinciding with $b=-0.6$ in Figure 4. Then follows, since the curve with exponents $b$ and -0.6 subjectively cross the margin at the same time:

$$
\frac{b_{1}}{\ln \alpha_{1}+b_{1} \beta_{1}}=\frac{-0.6}{\ln \alpha_{2}-0.6 \beta_{2}}
$$

Similarly, we assume an ascending function with an exponent $b_{2}$ such that $b_{2}$ coincides with $b=-0.2$ in Figure 4. Then follows again:

$$
\frac{b_{2}}{\ln \alpha_{1}+b_{2} \beta_{1}}=\frac{-0.2}{\ln \alpha_{2}-0.2 \beta_{2}}
$$

From Equations 9 and 10, we derive

$$
\beta_{2}-\beta_{1}=2.5 \ln \alpha_{1}\left(\frac{0.6}{b_{1}}-\frac{0.2}{b_{2}}\right) \text {. }
$$

Table 3

Dominance Matrix After Rearrangement of Rows and Columns

\begin{tabular}{ccccccc}
\hline & .2 & .4 & .1 & .3 & .6 & .5 \\
\hline-.2 & 0 & 0 & 0 & 1 & 1 & 1 \\
-.1 & 0 & 0 & 1 & 1 & 1 & 1 \\
-.3 & 0 & 1 & 1 & 1 & 1 & 1 \\
-.5 & 1 & 1 & 1 & 1 & 1 & 1 \\
-.4 & 1 & 1 & 1 & 1 & 1 & 1 \\
-.6 & 1 & 1 & 1 & 1 & 1 & 1 \\
\hline
\end{tabular}

Hence $\beta$ is improved when $\alpha_{1}>1$ and $b_{2} / b_{1}>1 / 3$ (or when $1>\alpha_{1}>0$ and $b_{2} / b_{1}<1 / 3$ ). It is suggested by Figure 4 that $b_{2} / b_{1}>1 ;\left(0.6 / b_{1}-0.2 / b_{2}\right)=1.5$ is only a conservative estimate. Since $\alpha_{1}=1.1$ and $\beta_{1}=0.0$ are not unusual outcomes for curves with positive b (Wagenaar \& Sagaria, 1975, Figure 5), we may estimate some minimal value of $\beta_{2}: \beta_{2}>2.5$ (0.1) $(1.5)=0.37$. Although the misperception of exponential growth in graphs occurs much less when graphs are plotted inversely, the gain is not as impressive as in the case of numerical presentations. The reason for the improvement might be found in a factor mentioned before: The major part of the trajectory between initial and final values is already covered by the part of the curve presented to the subject. Negative exponents make the process reveal its most tricky curvature in the stimulus part.

\section{REFERENCES}

DAWES, R. M.Fundamentals of attitude measurement. New York: Wiley, 1972.

JoNES, G. V.Polynomial perception of exponential growth. Perception \& Psychophysics, 1977, 21, 197-198.

SIEGEL, S. Nonparametric statistics for the behavioral sciences. New York: McGraw-Hill, 1956.

TVersky, A., \& Kahneman, D. Availability: A heuristic for judging frequency and probability. Cognitive Psychology, 1973, 5, 207-232.

WagenaAR, W. A., \& SAgaria, S. Misperception of exponential growth. Perception \& Psychophysics, 1975, 18, 416-422.

(Received for publication November 9, 1976; revision accepted March 28, 1977.) 